\title{
The impact of COVID-19 and strategies for mitigation and suppression in low- and middle-income countries
}

\author{
Patrick G. T. Walker ${ }^{1 *}+$, Charles Whittaker' ${ }^{1}$, Oliver J Watson ${ }^{1,2} \uparrow$, Marc Baguelin $^{1,3}$, Peter Winskill ${ }^{1}$, Arran \\ Hamlet ${ }^{1}$, Bimandra A. Djafaara ${ }^{1}$, Zulma Cucunubá ${ }^{1}$, Daniela Olivera Mesa', Will Green', Hayley Thompson', \\ Shevanthi Nayagam', Kylie E. C. Ainslie ${ }^{1}$, Sangeeta Bhatia ${ }^{1}$, Samir Bhatt ${ }^{1}$, Adhiratha Boonyasiri ${ }^{1}$, Olivia Boyd ${ }^{1}$, \\ Nicholas F. Brazeau', Lorenzo Cattarino', Gina Cuomo-Dannenburg'1, Amy Dighe ${ }^{1}$, Christl A. Donnelly ${ }^{1,4}$, Ilaria \\ Dorigatti $^{1}$, Sabine L. van Elsland ${ }^{1}$, Rich FitzJohn' ${ }^{1}$, Han Fu' ${ }^{1}$, Katy A.M. Gaythorpe ${ }^{1}$, Lily Geidelberg', Nicholas \\ Grassly $^{1}$, David Haw ${ }^{1}$, Sarah Hayes ${ }^{1}$, Wes Hinsley ${ }^{1}$, Natsuko Imai ${ }^{1}$, David Jorgensen ${ }^{1}$, Edward Knock ${ }^{1}$, Daniel

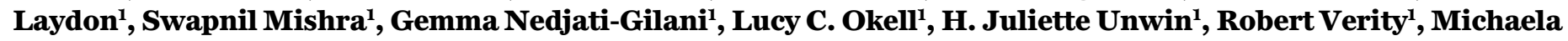 \\ Vollmer', Caroline E. Walters', Haowei Wang', Yuanrong Wang ${ }^{1}$, Xiaoyue Xi', David G Lalloo ${ }^{5}$, Neil M. \\ Ferguson ${ }^{1 *}$, Azra C. Ghani* \\ ${ }^{1}$ MRC Centre for Global Infectious Disease Analysis, Department of Infectious Disease Epidemiology, Imperial College London, London, UK. ²Pathology and Laboratory \\ Medicine, Warren Alpert Medical School, Brown University, Providence, RI, USA. ${ }^{3}$ Department of Infectious Disease Epidemiology, London School of Hygiene and Tropical \\ Medicine, London, UK. ${ }^{4}$ Department of Statistics, University of Oxford, Oxford, UK. ' Liverpool School of Tropical Medicine, Liverpool, UK. \\ *Corresponding author. E-mail: patrick.walker@imperial.ac.uk (P.G.T.W.); a.ghani@imperial.ac.uk (A.C.G.); neil.ferguson@imperial.ac.uk (N.M.F.) \\ †These authors contributed equally to this work.
}

The ongoing COVID-19 pandemic poses a severe threat to public health worldwide. We combine data on demography, contact patterns, disease severity, and health care capacity and quality to understand its impact and inform strategies for its control. Younger populations in lower income countries may reduce overall risk but limited health system capacity coupled with closer inter-generational contact largely negates this benefit. Mitigation strategies that slow but do not interrupt transmission will still lead to COVID-19 epidemics rapidly overwhelming health systems, with substantial excess deaths in lower income countries due to the poorer health care available. Of countries that have undertaken suppression to date, lower income countries have acted earlier. However, this will need to be maintained or triggered more frequently in these settings to keep below available health capacity, with associated detrimental consequences for the wider health, well-being and economies of these countries.

The COVID-19 pandemic caused by the SARS-CoV-2 virus is a major global health threat, with 5.4 million cases and 344,000 deaths confirmed worldwide as of the $26^{\text {th }}$ May 2020 (1). The experience in countries to date has emphasized the intense pressure that a COVID-19 epidemic places on national health systems, with demand for intensive care beds and mechanical ventilators rapidly outstripping their availability, even in relatively highly resourced settings (2). This has potentially profound consequences for resource-poor settings where the quality and availability of health care and related resources (such as oxygen) is typically poorer (3). We sought to understand the factors that could result in a differential impact of the COVID-19 pandemic in low- and middle-income countries (LMICs) as well as to evaluate the potential strategies for suppression and mitigation in these settings given the current global state of the pandemic.

\section{Demography and social contact patterns}

We collated data on global demographic projections of population size by age and country and available data on social mixing patterns by age and country-level income category.
We first include these within a simple SIR modelling framework (4) to estimate the theoretical final size of the outbreak (age-specific attack rate) in the absence of non-pharmaceutical interventions (NPIs). To illustrate how these would determine the demand for health care over the course of an unmitigated epidemic we applied age-specific estimates of the rates of hospitalization and of the proportion of these requiring critical care, and of the infection fatality ratio (IFR) (5) under an initial assumption of a consistent underlying role of comorbidities and the same level of medical care supplied during the epidemic in China (see Materials and Methods). On the basis of the observed doubling time in the incidence of deaths across Europe (6), we use a central estimate of the basic reproduction number $\left(\mathrm{R}_{0}\right)$ of 3.0 (a 3.5 day doubling time) and investigate scenarios with $\mathrm{R}_{0}$ between 2.3 (a 5 day doubling time) and 3.5 (a 3 day doubling time).

Figure 1 summarizes two of the demographic and societal factors which are likely to determine the burden of COVID19 disease across different income settings. First, there is a strong correlation between the gross domestic product (GDP) of a country and its underlying demography (Fig. 1A). Higher 
income countries tend to have the oldest populations; lower income countries in contrast have a much smaller proportion of the population who are above 65 and therefore within the age interval currently observed to be at particularly high risk of mortality from COVID-19 disease (5). Second, the household is a key setting for SARS-CoV-2 transmission (7). The average size of households that have a resident over the age of 65 years is substantially higher in lower income countries (Fig. 1B) compared with middle- and high-income countries, increasing the potential for spread generally but also specifically to this particularly vulnerable age-group. Contact patterns between age-groups also differ by country (fig. S5); in high-income settings the number of contacts tends to decline steeply with age. This effect is more moderate in middle-income settings and disappears in low-income settings, indicating that elderly individuals in these settings (LICs and MICs) maintain higher contact rates with a wider range of agegroups compared to elderly individuals in high-income countries (HICs). These contact patterns influence the predicted SARS-CoV-2 infection attack rate across age-groups (Fig. 1, C to E) with higher attack rates in the elderly predicted in lowincome settings compared to high-income settings and middle-income settings showing intermediate patterns.

For an unmitigated epidemic, we obtain similar estimates of the distribution of the attack rate across settings for a given $R_{0}$ (Fig. $1 \mathrm{~F}$ ), with slightly higher attack rates in lower income countries due to the more homogeneous levels of mixing with age. However, under a baseline assumption of the same comorbidity profile across all settings, we would expect a lower risk of requiring hospitalization and critical care in lower income settings, driven by the younger demography in these populations (Fig. 1, G to H). Assuming the same availability of health care (equivalent to that provided in China) throughout the pandemic, we would expect a lower overall per-capita risk of mortality in lower income settings due to the younger age of the populations (Fig. 1I).

\section{Health care availability and quality}

It is clear from the current epidemics in Europe and the United States that COVID-19 disease will place a severe strain on health systems. This effect is likely to be more extreme in lower-income settings where health care capacity is typically limited. To explore this, using data derived from the World Bank and wider literature, we developed a model of the supply of health care relevant to COVID-19 disease. We use a boosted regression tree-based approach to model the likely availability of hospital beds (per 1000 population, from the World Bank) based on a suite of relevant health care-related and socio-economic covariates (also from the World Bank, see Materials and Methods). This prediction of hospital bed capacity was then combined with estimates of intensive care unit beds (per 100 hospital beds) across a range of different settings (spanning LICs, LMICs, UMICs and HICs) identified through a systematic literature review (Fig. 2). These estimates of health care capacity were then integrated with our estimates of the demand that COVID-19 epidemics will place on national health systems.

The boosted regression tree model predicts hospital bed capacity well across the range of countries for which data were available (Fig. 2A). We find that hospital bed capacity is strongly correlated with the income status of countries (Fig. 2B); LICs have the fewest hospital beds per 1000 population (median 1.28 beds per 1000) and HICs the highest (median 4.68 beds per 1000 population). Lower and upper middle-income countries (LMIC/UMICs) fall between these two extremes (1.77 and 2.63 beds per 1000 population on average, respectively). We find that the percentage of hospital beds that are in intensive care units (ICU) is lowest in LICs (1.47\% on average) and highest in HICs (3.30\%) with LMICs and UMICs falling in-between $(2.00 \%$ and $2.88 \%$ respectively) (Fig. 2C). Note that our estimates of the ICU capacity in HICs are drawn almost exclusively from a recent review of ICU capacity in Asian countries (8) and are not therefore necessarily reflective of ICU capacity in HICs worldwide.

To understand the potential impact of weaker health systems on the IFR, we collated expert clinical opinion on the likely outcomes for COVID-19 patients (Materials and Methods). These expert estimates drew on recent experience treating COVID-19 patients in the UK alongside an understanding of severe pneumonia outcomes in LIC and LMIC settings. Mechanical ventilation (MV) - which has been required by $80 \%$ of COVID-19 ICU patients in the UK (9)- is of very limited capacity in many LIC and LMIC countries - across sub-Saharan Africa for example, recent estimates put the average number of ventilators at only 172 per country (10).. In the absence of MV, the consensus was that the mortality rate would be in the range $90-100 \%$. This compares to a mortality rate of $51.6 \%$ in those that require MV in the UK (9). Similarly, for the $20 \%$ of individuals that would be admitted to ICU in the UK but not require MV, the consensus was that mortality would be $50-65 \%$ in an LIC/LMIC setting. It was however noted that there would likely be significant heterogeneity in this rate (not captured here) due to the variation in both the quality of hospital care and availability of hospital facilities within and between countries (with better facilities concentrated in urban areas and capital cities compared to rural areas). For those with severe pneumonia requiring hospitalization, mortality rates are expected to be higher in LICs and LMICs than in HICs. The values for LICs and LMICs assumed here are a mortality rate across all age-groups of 20$30 \%$ if oxygen support is available while anticipating that this may not be at sufficiently high-flow directly at the bedside to ensure comparable outcomes to HIC ("Poorer Outcomes" in Fig. 2) and $60 \%$ if oxygen support is not available (due to 
health care capacity being exceeded). Using these parameters, we expect a larger proportion of deaths to occur in those aged 40 and upwards in LIC and LMIC settings (Fig. 2D) and that the lack of quality oxygen support will disproportionately increase mortality in younger age-groups (as these agegroups are more likely to require oxygen support than mechanical ventilation). Importantly, the lack of health system capacity is likely to increase the overall IFR in LIC and LMIC settings, off-setting the apparent protective effects of the younger population (Fig. 2E). In our subsequent modelling, we therefore examined three scenarios for LICs/LMICs to examine the impact of health care quality and quantity upon potential COVID-19 burden: a scenario where there were no health care constraints and quality of care was similar to HICs ("Unlimited health care"), a scenario using typical health care constraints on hospital and ICU beds in Fig. 2, B and C ("Limited health care") and a scenario where there is an assume absence of mechanical ventilation and treatment for severe pneumonia is less effective ("Limited health care, No MV and poorer outcomes").

\section{Co-morbidities}

There remain large uncertainties in the underlying determinants of the severity of SARS-CoV-2 infection and how these translate across settings. However, clear risk factors include age (5) and underlying co-morbidities that include hypertension, diabetes, coronary vascular disease (CVD) and chronic obstructive pulmonary disease (COPD) which serve to exacerbate symptoms (11). The prevalence of these conditions varies substantially across populations and by age (Fig. 3). Using Global Burden of Disease 2017 estimates (12), our unmitigated scenario leads to $6.1 \%, 3.8 \%$ and $13.3 \%$ of SARS-CoV-2 infections occurring in individuals with CVD, COPD and diabetes respectively.

In low-income (LIC) and low-middle income countries (LMIC) there is a higher burden of infectious diseases such as HIV/AIDS and TB, and of poverty-related determinants of poorer health outcomes such as malnutrition than in HICs. These generally occur in younger populations (Fig. 3, D to F). While infectious diseases and malnutrition are not yet recognized as specific risk-factors for poor prognosis from COVID-19 due to a lack of data from settings in which they are prevalent, it is possible that the risk profile in LIC and LMIC settings will be very different from that observed to date in China, Europe and North America. Understanding the extent to which these potential comorbidities make younger populations more vulnerable to severe sequalae of COVID-19 and designing strategies to protect them will be important in adapting pandemic responses to lower income countries.

\section{Mitigation and suppression strategies for low- and mid- dle-income countries}

To understand the consequences of the demographic, social contact and health system patterns on strategies for reducing the spread of SARS-CoV-2 in the context of control measures already enacted by countries, we developed an age-structured SEIR modelling framework to explore the dynamics of the epidemic under different health system capacity constraints (see Materials and Methods). As for the SIR final size calculations, we use demographic data and social contact patterns based on representative settings in the LIC, LMIC, UMIC and HIC strata. Disease progression for those requiring hospitalization was explicitly modeled in order to track requirements for hospital bed provision for severe pneumonia (for whom we assume oxygen support will be required) and for ICU provision (for whom we assume $80 \%$ would require MV in-line with UK data (9)). Durations of stay were based on UK data $(9,13)$; while it is likely that these will be shorter in LIC and LMIC countries, in the absence of data to guide these parameters the results presented here are conservative (i.e., hospital capacity will be exceeded earlier). While health care seeking behavior may also differ across settings, we make the simplifying assumption that all symptomatic cases seek care. To capture the uncertainty in hospital demand we generated 500 parameter sets drawing on uncertainty in key parameters determining the probability of hospitalization, of requiring critical care, and of outcomes (death or recovery) in each hospital state and ran 10 stochastic realization using each parameter set (see Materials and Methods). The 95\% range across the simulations is presented as an uncertainty interval (UI). We consider two potential strategies (similar to those previously illustrated for pandemic influenza planning and in the early stages of the COVID-19 epidemic $(14,15))$; (1) mitigation whereby transmission is reduced but $\mathrm{R}_{\mathrm{t}}$ remains above 1 and hence a single-peaked epidemic is predicted due to the buildup of herd immunity; and (2) suppression - whereby transmission is reduced such that $\mathrm{R}_{\mathrm{t}}<1$ and hence, if interventions are later released, transmission will be expected to rise as herd immunity will not have been achieved.

We used our final SIR size calculations to estimate the degree of social distancing that results in "optimal" mitigation. This is defined as the maximum reduction in transmission that can be achieved if a uniform reduction in contact rates is implemented at the start of the epidemic for an undefined but finite period such that $\mathrm{R}_{\mathrm{t}} \sim 1$ and a single-peaked epidemic is generated (see Materials and Methods). If "optimal" mitigation based on enhanced social distancing is pursued, for an $\mathrm{R}_{0}$ of 3.0, we estimate a maximum reduction in infections in individual countries in the range $30-38 \%$ (median $33 \%$ ) and a range of reduction in mortality between $19 \%-55 \%$ (median $39 \%$ ) (assuming the mortality patterns observed in China). These optimal reductions in transmission and burden were achieved with a range of reductions in the overall rate of social contact across countries between $40.0 \%-44.9 \%$ (median $43.9 \%$ ), with this range across countries increasing to $42.9 \%$ $47.9 \%$ (median $46.9 \%$ ) for an $\mathrm{R}_{0}$ of 3.5 and decreasing to 
$31.4 \%-35.8 \%$ across countries (median $35.0 \%$ ) for an $\mathrm{R}_{0}$ of 2.3 . Combining mitigation with enhanced social distancing of elderly individuals is predicted to result in greater mortality reductions of $23 \%-67 \%$ across countries (median $49 \%$ ) for $\mathrm{R}_{0}=3$ (table $\mathrm{S} 5$ ). However, both of these strategies are predicted to have lower proportional impact in lower income settings compared to higher income settings: median reductions in mortality in the range $19.5 \%-41.6 \%$ (median $25.3 \%$ ) in LICs in contrast to in the range $21.5 \%-55.1 \%$ (median $49.9 \%$ ) in HICs for optimized mitigation strategies including social distancing and in the range $25.4 \%-50.9 \%$ (median $32.6 \%$ ) in LICs in contrast to in the range $23.4 \%-66.6 \%$ (median $60.1 \%$ ) in HICs for optimized mitigation strategies including enhanced social distancing for elderly (table S5). This lower proportional reduction in deaths of mitigation scenarios in lower income settings is driven by the more homogeneous contact patterns by age in these settings (Fig. 1), resulting in more persistent spread to older age categories as contact rates in the general population are reduced (fig. S6).

Figure 4 highlights the dynamical impact of different control measures on COVID-19 epidemics. Scenarios in which in which the COVID-19 epidemic is suppressed for a period of six months before returning to pre-pandemic social contact patterns leads to rapid resurgence of the virus and a delayed peaking epidemic (Fig. 4A). There is significant reduction in disease burden under the optimal mitigation strategy, which results in a single-peaked epidemic with a substantially lower peak compared to the unmitigated epidemic. It should be noted, however, that this single peak relies upon the assumption that recovery from infection confers durable immunity to reinfection which has yet to have been conclusively demonstrated (16). Furthermore, while "optimal" mitigation is the strategy that will minimize infections and achieve herd immunity in a single-peaked epidemic, there are multiple other strategies that can also minimize infections over a longerterm $(17,18)$. Any mitigation scenario will also always be worse in terms of both the peak hospital demand and total predicted deaths than scenarios in which the epidemic is suppressed (i.e., the reproduction number over time, $R_{t}$, is kept below 1) (Fig. 4, A and B). However, if suppression cannot be successfully maintained, then a delayed epidemic may occur which may outweigh the benefits of the original suppression strategy and result in higher mortality than if a mitigation scenario had been successfully pursued. Such a second peak is not inevitable - widescale suppression may provide countries with the time to develop testing and contact tracing systems, as well as locally targeted responses, that can help to maintain lower levels of transmission once the initial suppression interventions are relaxed. It is important to note that our framework does not currently provide insights into the specific combinations of NPIs required to achieve such reductions and these are likely to differ across settings according to various factors such as school attendance and occupational factors. Other factors such as reduced ability to work from home and general economic vulnerability will impact on the abilities of populations to adhere to stringent NPIs that involve restrictions in movement. Meanwhile, larger household sizes, and subsequently higher levels of household-based transmission, may limit the impact of selfisolation and increase the social and economic impact of selfisolation measures in lower income settings.

The comparative benefits and drawbacks of these scenarios (in terms of direct health impact of COVID-19 disease) will differ between settings depending on their health care capacity and quality. In all settings, while our optimized mitigation scenario is predicted to substantially reduce the gap between demand for hospital beds and capacity, demand for critical care is still predicted to vastly exceed capacity, leading to a substantial additional burden relative to a scenario with unlimited capacity (Fig. 4B). Although we predict lower demand for critical care in lower income settings due to their younger populations, this is likely to be offset by a much lower level of supply: for our mitigation scenario including populationlevel social distancing, peak demand for critical care in our simulation for a typical LIC outstrips supply by a factor of 30.7 (95\% UI, 14.7 - 48.8), whereas for the equivalent simulation in a typical HIC this factor was 7.8 (95\% UI 3.6 - 13.0) (Fig. 4C). Typical LMIC and UMIC produced factors of overdemand of 17.5 (95\% UI 8.3 - 28.6) and 10.9 (95\% UI 5.1 - 17.7) respectively.

We estimate that constraints on health care capacity would be likely to increase the IFR under a mitigation strategy in all settings. However, as has been observed, HICs and UMICs are likely to be able to put in place surge capacity to limit any impact on mortality (19-21). In contrast, in LICs and LMICs we predict that the poorer quality of health care available is likely to have a greater impact on the overall IFR than the limits on capacity alone. If the health care quality in these settings was at the same level as in HICs and not subject to capacity constraints, we estimate $2.1(95 \%$ UI 1.0-3.3) deaths per 1,000 population in an LIC and 2.4 (95\% UI 1.1-3.9) deaths per 1,000 population in an LMIC. This increases to $4.6(95 \%$ UI 1.4-10.0) and 3.9 (95\% UI 1.5-8.9) deaths per 1,000 population with health care capacity limits, in LICs and LMICs respectively, and to 6.5 (95\% UI $2.7-12.3)$ and $6.2(95 \%$ UI 2.8 11.9) deaths per 1,000 population, respectively, if the poorer quality health care is also factored in. Overall, this represents 4.4 (95\% UI 1.7-9.0) and 3.8 (95\% UI 1.7-8.0) excess deaths per 1,000 population due to both the poorer quality health care and lack of health care capacity in LICs and LMICs respectively.

\section{Suppression and longer-term exit strategies}

Almost all countries and territories have now reported at 
least one COVID-19 case with many now also reporting deaths. While individual countries have responded differently to this threat, the majority have implemented some form of NPI to either mitigate the burden of the epidemic or to suppress transmission $(22,23)$. We reviewed data on the interventions that had been collated within the ACAP COVID-19 Government Response Measures dataset to summarize the stage of the epidemic at which countries have implemented suppression measures (23) (see Materials and Methods).

Table 1 summarizes the stage of the epidemic at which those countries implementing suppression measures did so (see Supporting Material for country-level analysis). Across the different regions, countries in Europe and Central Asia have initiated suppression measures at a later stage of their epidemics (in terms of per-capita cases and deaths) than other regions to date. This may partly be due to censoring (i.e., other countries have yet to impose suppression measures since the date the dataset was downloaded - April $20^{\text {th }} 2020$ ) as well as due to the wider recognition of the potential impact of COVID-19 that countries in other regions can observe from the ongoing epidemics in Europe. However, there is also a strong gradient in the timing of lockdowns with income status - with LIC and LMIC initiating suppression measures earlier than UMIC or HIC.

We used European Centre for Disease Control (ECDC) data prior to the date of implementation of suppression or, in the absence of identified suppression measures, the date of last entry within the ACAPS dataset. We then evaluated the ratio of reported cases to deaths in this period to provide a measure of the capacity of countries to contain transmission through testing-based approaches prior to, or in the absence of, suppression. Our estimates show clear differences by region and gradient across income strata: LICs with three or more deaths prior to suppression reported a country-level median of 8.6 cases per reported death (country-level range of 7.8 - 10.7, $\mathrm{n}=3$ ) and LMICs a country-level median of 19.3 (country-level range $9-80, \mathrm{n}=7$ ). In contrast, HICs reported a country-level median of 72.6 (country-level range $9-325.2$, $\mathrm{n}=27$ ) cases per reported death. The extent to which reported case-to-death ratios are a reliable indicator of relative case or infection (including asymptomatic) ascertainment rates will depend upon trends in IFR, the reproduction number and the extent to which deaths are reported, all of which are liable to vary across income strata. However, this trend is suggestive of the extent to which testing capacity will need to be developed in LICs/LMICs settings if approaches such as case identification coupled with contact tracing are to form part of a successful mitigation or exit strategy.

Given our estimates suggest that even optimal mitigative strategies will lead to substantial excess mortality and exceedance of health care capacity, we explored the impact of potentially different suppressive strategies, accounting for the interventions that countries have implemented to date. Here we define suppression as reducing transmission to a level for which $R_{t}<1$. To do so we model a $75 \%$ reduction in contact rates across all age-groups, giving $R_{t}=0.75$ for $R_{0}=3.0$. We explored different trigger thresholds based on the incidence of cases requiring critical care per 100,000 population) for the implementation of transmission reductions and modeled these as lasting for 30 days before being lifted (typical of the duration of lockdowns occurring to date). Reimplementation then occurs if the trigger threshold is eclipsed again. The level of reduction in contact rates will determine the speed at which the infected population is depleted during the intervention (24). Thus, for a fixed period of intervention (assumed here to be 1 month) either starting earlier (i.e., at lower levels of infection) or suppressing to a greater extent (i.e., higher reductions in contact rates) will mean that interventions can be relaxed for longer before the trigger for reimplementation is reached (Fig. 5A). Equally, if a degree of suppression is maintained during the period of relaxation (for example, a $30 \%$ reduction in contacts during relaxation compared to $75 \%$ during suppression), then the periods in suppression will be shorter as the reduced $R_{t}$ during relaxation will mean that it takes longer to trigger the suppression threshold. Furthermore, a greater health benefit (in terms of reducing cases or deaths) will be achieved for lower trigger levels; however, this is balanced by a slower build-up of herd immunity such that the interventions would need to remain in place for longer in the absence of a vaccine (Fig. 5B).

To further understand how such a strategy might differ between LIC/LIMC and UMIC/HIC we explored the full range of incidence thresholds that would allow ICU demand to be kept below $50 \%$ of the median ICU capacity for each income strata (setting this threshold lower than maximum capacity to allow for ongoing care provision for non-COVID-19-related disease). For each scenario, we modeled the first suppression to have been initiated at the median threshold for the setting that has been observed to date (i.e., LIC and LMIC initiate earlier than UMIC and HIC, Table 1). We then selected the ICU incidence trigger within this range that minimizes COVID-19 mortality over 18 months and remains under our ICU threshold. We use a time window of 18 months as representative of the timescale over which pharmaceutical interventions (e.g., a vaccine) may become available, noting that this duration is highly uncertain (25).

If ICU demand is to be kept below the estimated median ICU capacity for each income strata, all countries are predicted to need to spend a substantial proportion of time in suppression (Fig. 5C). In all settings, in the absence of additional effective measures, we estimate that the time spent in suppression to prevent health services becoming overwhelmed will need to be high across all settings, and 
marginally higher in LICs (77\% vs $66 \%$ in HICs over an 18 month timeframe) driven by the lower threshold at which suppression has to be reapplied coupled with the resulting less rapid acquisition of immunity. Assuming identical quality but not quantity of care across all settings, the mortality rate under suppression is predicted to be highest in HICs across all suppression threshold triggers considered (and by extension, time in suppression over the next 18 months) due to their older populations (Fig. 5D, purple lines). However, once we incorporate our estimates of the poorer quality of care in LICs and LMICs we predict a similar number of deaths in LIC and LMIC compared to HICs across all suppression triggers. Thus, in all settings, sustained periods of suppression over the next 18 months are predicted to be required if ICU demand is to be kept below capacity and large levels of excess mortality are to be averted. Conversely, we also estimate that the risks of not maintaining suppression are likely to be similarly high across all settings. If suppression is not maintained (and hence the proportion of time spent in lockdown over the next 18 months is low), then our results suggest a lower per-capita level of burden LIC/LMIC due to the younger population. However, the uncertainty in our estimates of the quality of care in these settings could mean that this result is reversed (Fig. 5D).

Our estimates of increasing time between suppression triggers in HICs in Fig. 5E assume that there is durable immunity to reinfection - this remains uncertain (16). However, given the very low levels of population immunity in LIC/LMICs at 18 months in the presence of this assumption, our results indicate that in these settings measures would have to remain in place well beyond the time window of our simulations in the absence of a vaccine in order to achieve herd immunity, or equivalent effective exit strategy able to maintain control of the epidemic for values of $R_{t}$ that remain close to $\mathrm{R}_{0}$.

Importantly, however, these results do not account for other interventions that could be implemented during periods where suppressive measures are not in place. Once the number of new infections drops to a manageable level, it is likely that more widespread testing and isolation of cases coupled with contact tracing can help to prevent a resurgence of transmission, as has been observed in countries such as South Korea (26-28). However, given the low reported case to reported death ratios in LICs/LMICs during the early stage of the pandemic, for such strategies to be successful it is likely that support to enhance surveillance will be required to increase infection ascertainment rates substantially. Such testing strategies could be supplemented by the additional use of technology including digital apps (29). The appropriateness of these different interventions will be context-specific and hence it is likely that each country will need to develop strategies based on an understanding of the underlying principles outlined here but adapted to suit their needs.

\section{Conclusions}

The results presented here illustrate the potential impact of the COVID-19 pandemic in LICs and LMICs compared to the epidemics that have occurred to date in UMICs and HICs. Our analyses give insight into how differences in demography, social structure and health care availability and quality combine and potentially influence the impact of measures that can help reduce the spread of the virus. At the current time, it is not possible to predict with any certainty the exact number of cases for any given country, the precise mortality and disease burden that will result or the benefits and drawbacks of the different approaches to control of the virus that are currently being implemented. A full understanding of these will only be available retrospectively.

While our results illustrate the challenges that many countries will face in attempting to mitigate the impact of local COVID-19 epidemics, it is important to bear in mind that even moderate levels of changes in behavior can avert many infections and hence save millions of lives (30). While suppression will always have the greatest impact on COVIDrelated morbidity and mortality, the intensity of interventions required needs to be balanced against the wider health risks that diverting all attention to a single disease could entail (31, 32).

It is also important to note that we do not quantify the wider societal and economic impact of the intensive mitigation or suppression approaches, nor address the challenge of intensive suppression initiatives in LIC and LIMC where a high degree of informal labor makes such interventions challenging and may limit the extent to which they can reduce $R_{t}$ below one (33). These are likely to be substantial, particularly in lower income countries where the capacity to provide support in ensuring the livelihoods of the poorest and most vulnerable is most marginal. Moreover, for countries lacking the infrastructure capable of implementing technology-led suppression strategies such as those currently being pursued in Asia $(7,27)$, and in the absence of a vaccine or other effective therapy, careful thought will need to be given to pursuing such strategies in order to avoid a high risk of future health system failure once suppression measures are lifted.

Our results highlight the difficult decisions countries are faced with in the coming weeks and months irrespective of region or income status. Given the likely worse prognosis of severe COVID-19 cases in settings with weaker health systems coupled with the higher vulnerability of developing economies to the negative effects of stringent NPIs, the trade-offs lower income countries face are complex given the ongoing uncertainty in the most appropriate and effective exit strategies. In the interim, the priority should be to increase the availability of oxygen support to mitigate the health impact 
alongside enhancing the capacity for surveillance and widescale testing to reduce the spread of infection and tailor appropriate NPIs. In the longer-term, ensuring equitable provision of pharmaceutical interventions to lower income countries once they are developed should be a global priority.

Our analysis demonstrates the extent to which countries have mobilised to combat the COVID-19 pandemic. Many lower income countries have acted while transmission remains at low levels which is likely to have substantially slowed the spread of the virus. In the absence of a vaccine, all governments are likely to face challenging decisions around intervention strategies for the foreseeable future. However, the still relevant counterfactual of a largely unmitigated pandemic clearly demonstrates the extent to which rapid, decisive and collective action remains critical to save lives globally.

\section{REFERENCES AND NOTES}

1. WHO, "Coronavirus disease (COVID-19) Situation Report-127" (2020); www.who.int/docs/default-source/coronaviruse/situation-reports/20200526covid-19-sitrep-127.pdf?sfursn=7b6655ab_8.

2. A. Remuzzi, G. Remuzzi, COVID-19 and Italy: What next? Lancet 395, 1225-1228 (2020). doi:10.1016/S0140-6736(20)30627-9 Medline

3. A. S. Ginsburg, W. C. Van Cleve, M. I. W. Thompson, M. English, Oxygen and pulse oximetry in childhood pneumonia: A survey of healthcare providers in resourcelimited settings. J. Trop. Pediatr. 58, 389-393 (2012). doi:10.1093/tropej/fmr103 Medline

4. J. Cui, Y. Zhang, Z. Feng, Influence of non-homogeneous mixing on final epidemic size in a meta-population model. J. Biol. Dyn. 13 (sup1), 31-46 (2019). doi:10.1080/17513758.2018.1484186 Medline

5. R. Verity, L. C. Okell, I. Dorigatti, P. Winskill, C. Whittaker, N. Imai, G. CuomoDannenburg, H. Thompson, P. G. T. Walker, H. Fu, A. Dighe, J. T. Griffin, M. Baguelin, S. Bhatia, A. Boonyasiri, A. Cori, Z. Cucunubá, R. FitzJohn, K. Gaythorpe, W. Green, A. Hamlet, W. Hinsley, D. Laydon, G. Nedjati-Gilani, S. Riley, S. van Elsland, E. Volz, H. Wang, Y. Wang, X. Xi, C. A. Donnelly, A. C. Ghani, N. M. Ferguson, Estimates of the severity of coronavirus disease 2019: A model-based analysis. Lancet Infect. Dis. 20, 669-677 (2020). doi:10.1016/S14733099(20)30243-7 Medline

6. S. Flaxman, S. Mishra, A. Gandy, J. T. Unwin, H. Coupland, T. A. Mellan, et al., Estimating the number of infections and the impact of non-pharmaceutical interventions on COVID-19 in 11 European countries (Imperial College COVID-19 Response Team) (2020); https://doi.org/10.25561/77731.

7. WHO, Report of the WHO-China Joint Mission on Coronavirus Disease, 2019 (COVID-19) (2020); www.who.int/publications-detail/report-of-the-who-chinajoint-mission-on-coronavirus-disease-2019-(covid-19).

8. J. Phua, M. O. Faruq, A. P. Kulkarni, I. S. Redjeki, K. Detleuxay, N. Mendsaikhan, K. K. Sann, B. R. Shrestha, M. Hashmi, J. E. M. Palo, R. Haniffa, C. Wang, S. M. R. Hashemian, A. Konkayev, M. B. Mat Nor, B. Patjanasoontorn, K. M. K. Nafees, L. Ling, M. Nishimura, M. J. Al Bahrani, Y. M. Arabi, C.-M. Lim, W.-F. Fang; Asian Analysis of Bed Capacity in Critical Care (ABC) Study Investigators, and the Asian Critical Care Clinical Trials Group, Critical Care Bed Capacity in Asian Countries and Regions. Crit. Care Med. 48, 654-662 (2020). doi:10.1097/CCM.0000000000004222 Medline

9. Intensive Care National Audit \& Research Centre (ICNARC), ICNARC report on COVID-19 in critical care 10 April 2020 (2020); www.icnarc.org/About/LatestNews/2020/04/04/Report-On-2249-Patients-Critically-ll-With-Covid-19.

10. K. Houreld, D. Lewis, R. McNeill, S. Granados, Virus exposes gaping holes in Africa's health systems. Reuters (2020); https://graphics.reuters.com/HEALTHCORONAVIRUS/AFRICA/yzdpxogbdvx/.

11. F. Zhou et al., Articles Clinical course and risk factors for mortality of adult inpatients with COVID-19 in Wuhan, China : A retrospective cohort study. Lancet $6736,1-9(2020)$.
12. S. L. Jameset al., Global, regional, and national incidence, prevalence, and years lived with disability for 354 diseases and injuries for 195 countries and territories, 1990-2017: A systematic analysis for the Global Burden of Disease Study 2017. Lancet 392, 1789-1858 (2018). doi:10.1016/S0140-6736(18)32279-7 Medline

13. A. B. Docherty, E. M. Harrison, C. A. Green, H. E. Hardwick, R. Pius, L. Norman, et al., Features of 16,749 hospitalised UK patients with COVID-19 using the ISARIC WHO Clinical Characterisation Protocol. medRxiv 2020.04.23.20076042 [Preprint]. 28 April 2020. https://doi.org/10.1101/2020.04.23.20076042

14. T. D. Hollingsworth, D. Klinkenberg, H. Heesterbeek, R. M. Anderson, Mitigation strategies for pandemic influenza A: Balancing conflicting policy objectives. PLOS Comput. Biol. 7, e1001076 (2011). doi:10.1371/journal.pcbi.1001076 Medline

15. R. M. Anderson, H. Heesterbeek, D. Klinkenberg, T. D. Hollingsworth, How will country-based mitigation measures influence the course of the COVID-19 epidemic? Lancet 395, 931-934 (2020). doi:10.1016/S0140-6736(20)30567-5 Medline

16. D. M. Altmann, D. C. Douek, R. J. Boyton, What policy makers need to know about COVID-19 protective immunity. Lancet 395, 1527-1529 (2020). doi:10.1016/S0140-6736(20)30985-5 Medline

17. D. H. Morris, F. W. Rossine, J. B. Plotkin, S. A. Levin, Optimal, near-optimal, and robust epidemic control (2020); https://arxiv.org/abs/2004.02209.

18. F. Di Lauro, I. Z. Kiss, J. Miller, medRxiv 2020.03.02.20030007 [Preprint]. 6 March 2020. https://doi.org/10.1101/2020.03.02.20030007.

19. L. Carenzo, E. Costantini, M. Greco, F. L. Barra, V. Rendiniello, M. Mainetti, R. Bui, A. Zanella, G. Grasselli, M. Lagioia, A. Protti, M. Cecconi, Hospital surge capacity in a tertiary emergency referral centre during the COVID-19 outbreak in Italy. Anaesthesia 75, 928-934 (2020). doi:10.1111/anae.15072 Medline

20. A. Zangrillo et al., Fast reshaping of intensive care unit facilities in a large metropolitan hospital in Milan, Italy: facing the COVID-19 pandemic emergency. Crit. Care Resusc. (2020); www.ncbi.nlm.nih.gov/pubmed/32227819.

21. E. Litton, T. Bucci, S. Chavan, Y. Y. Ho, A. Holley, G. Howard, S. Huckson, P. Kwong, J. Millar, N. Nguyen, P. Secombe, M. Ziegenfuss, D. Pilcher, Surge capacity of intensive care units in case of acute increase in demand caused by COVID-19 in Australia. Med. J. Aust. 212, 463-467 (2020). doi:10.5694/mja2.50596 Medline

22. T. Hale, S. Webster, A. Petherick, T. Phillips, B. Kira, "Oxford COVID-19 Government Response Tracker" (2020); www.bsg.ox.ac.uk/research/researchprojects/coronavirus-government-response-tracker.

23. ACAPS, \#COVID19 Government Measures Dataset I ACAPS (2020); www.acaps.org/covid19-government-measures-dataset.

24. N. M. Ferguson, D. Laydon, G. Nedjati-Gilani, N. Imai, K. Ainslie, M. Baguelin, et al., Impact of non-pharmaceutical interventions (NPIs) to reduce COVID-19 mortality and healthcare demand (Imperial College COVID-19 Response Team) (2020); https://doi.org/10.25561/77482.

25. N. Lurie, M. Saville, R. Hatchett, J. Halton, Developing Covid-19 Vaccines at Pandemic Speed. N. Engl. J. Med. 382, 1969-1973 (2020). doi:10.1056/NEJMp2005630 Medline

26. Consulate General of the Republic of Korea, "Flattening the curve on COVID-19 How Korea responded to a pandemic using ICT" (2020); http://overseas.mofa.go.kr/us-houston-en/brd/m 5573/view.do?seq=759765.

27. COVID-19 National Emergency Response Center, Epidemiology \& Case Management Team, Korea Centers for Disease Control \& Prevention, Contact Transmission of COVID-19 in South Korea: Novel Investigation Techniques for Tracing Contacts. Osong Public Health Res. Perspect. 11, 60-63 (2020).

28. K. E. C. Ainslie, C. E. Walters, H. Fu, S. Bhatia, H. Wang, X. Xi, M. Baguelin, S. Bhatt, A. Boonyasiri, O. Boyd, L. Cattarino, C. Ciavarella, Z. Cucunuba, G. CuomoDannenburg, A. Dighe, I. Dorigatti, S. L. van Elsland, R. FitzJohn, K. Gaythorpe, A. C. Ghani, W. Green, A. Hamlet, W. Hinsley, N. Imai, D. Jorgensen, E. Knock, D. Laydon, G. Nedjati-Gilani, L. C. Okell, I. Siveroni, H. A. Thompson, H. J. T. Unwin, R. Verity, M. Vollmer, P. G. T. Walker, Y. Wang, O. J. Watson, C. Whittaker, P. Winskill, C. A. Donnelly, N. M. Ferguson, S. Riley, Evidence of initial success for China exiting COVID-19 social distancing policy after achieving containment. Wellcome Open Res. 5, 81 (2020). doi:10.12688/wellcomeopenres.15843.1 Medline

29. L. Ferretti, C. Wymant, M. Kendall, L. Zhao, A. Nurtay, L. Abeler-Dörner, M. Parker, D. Bonsall, C. Fraser, Quantifying SARS-CoV-2 transmission suggests epidemic control with digital contact tracing. Science 368, eabb6936 (2020). doi:10.1126/science. abb6936 Medline 
30. P. G. Walker, C. Whittaker, O. Watson, M. Baguelin, K. E. C. Ainslie, S. Bhatia, et al., The Global Impact of COVID-19 and Strategies for Mitigation and Suppression (Imperial College COVID-19 Response Team) (2020); https://doi.org/10.25561/77735.

31. A. B. Hogan, B. Jewell, E. Sherrard-Smith, J. Vesga, O. J. Watson, C. Whittaker, et al., Report 19: The Potential Impact of the COVID-19 Epidemic on HIV, TB and Malaria in Low-and Middle-Income Countries, https://doi.org/10.25561/78670.

32. E. L. Korenromp, Lives saved from malaria prevention in Africa-Evidence to sustain cost-effective gains. Malar. J. 11, 94 (2012). doi:10.1186/1475-2875-11-94 Medline

33. N. V. Loayza, "Costs and Trade-Offs in the Fight Against the COVID-19 Pandemic : A Developing Country Perspective" (Washington, D.C, 2020); http://documents.worldbank.org/curated/en/799701589552654684/Costsand-Trade-Offs-in-the-Fight-Against-the-COVID-19-Pandemic-A-DevelopingCountry-Perspective.

34. 0. J. Watson et al., mrc-ide/squire, Version v.0.4.14, Zenodo (2020); https://doi.org/10.5281/zenodo.3872340.

35. C. Whittaker et al., covid_global_impact, Version 1.0, Zenodo (2020); https://doi.org/10.5281/zenodo.3872387.

36. 0. J. Watson, R. FitzJohn, J. W. Eaton, rdhs: An R package to interact with The Demographic and Health Surveys (DHS) Program datasets. Wellcome Open Res. 4, 103 (2019). doi:10.12688/wellcomeopenres.15311.1

37. Office for National Statistics, 2011 Census - household type, household size and age of usual residents (people) - England and Wales - (2018); www.ons.gov.uk/peoplepopulationandcommunity/housing/adhocs/008635ct0 8202011 censushouseholdtypehouseholdsizeandageofusualresidentspeopleengl andandwales?:uri=peoplepopulationandcommunity/housing/adhocs/008635ct 08202011censushouseholdtypehouseholdsiz.

38. T. Hoang, P. Coletti, A. Melegaro, J. Wallinga, C. G. Grijalva, J. W. Edmunds, P. Beutels, N. Hens, A Systematic Review of Social Contact Surveys to Inform Transmission Models of Close-contact Infections. Epidemiology 30, 723-736 (2019). doi:10.1097/EDE.0000000000001047 Medline

39. D. Moher, L. Shamseer, M. Clarke, D. Ghersi, A. Liberati, M. Petticrew, P. Shekelle, L. A. Stewart; PRISMA-P Group, Preferred reporting items for systematic review and meta-analysis protocols (PRISMA-P) 2015 statement. Syst. Rev. 4, 1 (2015). doi:10.1186/2046-4053-4-1 Medline

40. S. Kumar, M. Gosain, H. Sharma, E. Swetts, R. Amarchand, R. Kumar, K. E. Lafond F. S. Dawood, S. Jain, M. A. Widdowson, J. M. Read, A. Krishnan, Who interacts with whom? Social mixing insights from a rural population in India. PLOS ONE 13, e0209039 (2018). doi:10.1371/journal.pone.0209039 Medline

41. O. le Polain de Waroux, S. Cohuet, D. Ndazima, A. J. Kucharski, A. Juan-Giner, S. Flasche, E. Tumwesigye, R. Arinaitwe, J. Mwanga-Amumpaire, Y. Boum 2nd, F. Nackers, F. Checchi, R. F. Grais, W. J. Edmunds, Characteristics of human encounters and social mixing patterns relevant to infectious diseases spread by close contact: A survey in Southwest Uganda. BMC Infect. Dis. 18, 172 (2018). doi:10.1186/s12879-018-3073-1 Medline

42. J. Mossong, N. Hens, M. Jit, P. Beutels, K. Auranen, R. Mikolajczyk, M. Massari, S. Salmaso, G. S. Tomba, J. Wallinga, J. Heijne, M. Sadkowska-Todys, M. Rosinska, W. J. Edmunds, Social contacts and mixing patterns relevant to the spread of infectious diseases. PLOS Med. 5, e74 (2008). doi:10.1371/journal.pmed.0050074 Medline

43. G. Béraud, S. Kazmercziak, P. Beutels, D. Levy-Bruhl, X. Lenne, N. Mielcarek, Y Yazdanpanah, P.-Y. Boëlle, N. Hens, B. Dervaux, The French Connection: The First Large Population-Based Contact Survey in France Relevant for the Spread of Infectious Diseases. PLOS ONE 10, e0133203 (2015). doi:10.1371/journal.pone.0133203 Medline

44. K. Leung, M. Jit, E. H. Y. Lau, J. T. Wu, Social contact patterns relevant to the spread of respiratory infectious diseases in Hong Kong. Sci. Rep. 7, 7974 (2017). doi:10.1038/s41598-017-08241-1 Medline

45. J. Zhang, P. Klepac, J. M. Read, A. Rosello, X. Wang, S. Lai, M. Li, Y. Song, Q. Wei, H. Jiang, J. Yang, H. Lynn, S. Flasche, M. Jit, H. Yu, Patterns of human social contact and contact with animals in Shanghai, China. Sci. Rep. 9, 15141 (2019). doi:10.1038/s41598-019-51609-8 Medline

46. C. G. Grijalva, N. Goeyvaerts, H. Verastegui, K. M. Edwards, A. I. Gil, C. F. Lanata, N. Hens; RESPIRA PERU project, A household-based study of contact networks relevant for the spread of infectious diseases in the highlands of Peru. PLOS ONE 10, e0118457 (2015). doi:10.1371/journal.pone.0118457 Medline

47. M. Ajelli, M. Litvinova, Estimating contact patterns relevant to the spread of infectious diseases in Russia. J. Theor. Biol. 419, 1-7 (2017). doi:10.1016/i.jtbi.2017.01.041 Medline

48. S. P. Johnstone-Robertson, D. Mark, C. Morrow, K. Middelkoop, M. Chiswell, L. D. H. Aquino, L.-G. Bekker, R. Wood, Social mixing patterns within a South African township community: Implications for respiratory disease transmission and control. Am. J. Epidemiol. 174, 1246-1255 (2011). doi:10.1093/aje/kwr251 Medline

49. M. C. Kiti, T. M. Kinyanjui, D. C. Koech, P. K. Munywoki, G. F. Medley, D. J. Nokes, Quantifying age-related rates of social contact using diaries in a rural coastal population of Kenya. PLOS ONE 9, el04786 (2014). doi:10.1371/journal.pone.0104786 Medline

50. A. Melegaro, E. Del Fava, P. Poletti, S. Merler, C. Nyamukapa, J. Williams, S. Gregson, P. Manfredi, Social Contact Structures and Time Use Patterns in the Manicaland Province of Zimbabwe. PLOS ONE 12, e0170459 (2017). doi:10.1371/journal.pone.0170459 Medline

51. K. O. Kwok, B. Cowling, V. Wei, S. Riley, J. M. Read, Temporal variation of human encounters and the number of locations in which they occur: A longitudinal study of Hong Kong residents. J. R. Soc. Interface 15, 20170838 (2018). doi:10.1098/rsif.2017.0838 Medline

52. Y. Ibuka, Y. Ohkusa, T. Sugawara, G. B. Chapman, D. Yamin, K. E. Atkins, K. Taniguchi, N. Okabe, A. P. Galvani, Social contacts, vaccination decisions and influenza in Japan. J. Epidemiol. Community Health 70, 162-167 (2016) doi:10.1136/jech-2015-205777 Medline

53. International Monetary Fund, World Economic Outlook (2016); https://web.archive.org/web/20160421023851/http://www.imf.org/external/ pubs/ft/weo/2016/01/pdf/text.pdf.

54. Database of Global Administrative Areas; https://gadm.org/.

55. R. Hijmans, S. Phillips, J. Leathwick, J. Elith, dismo:Species Distribution Modeling. R package (2017); https://rspatial.org/raster/sdm/index.html.

56. S. Murthy, A. Leligdowicz, N. K. J. Adhikari, Intensive care unit capacity in lowincome countries: A systematic review. PLOS ONE 10, e0116949 (2015). doi:10.1371/journal.pone.0116949 Medline

57. European Centre for Disease Control, Situation update worldwide, as of 27 April 2020 (2020); www.ecdc.europa.eu/en/geographical-distribution-2019-ncovcases.

58. N. M. Linton, T. Kobayashi, Y. Yang, K. Hayashi, A. R. Akhmetzhanov, S. M. Jung, B. Yuan, R. Kinoshita, H. Nishiura, Incubation Period and Other Epidemiological Characteristics of 2019 Novel Coronavirus Infections with Right Truncation: A Statistical Analysis of Publicly Available Case Data. J. Clin. Med. 9, 538 (2020). doi:10.3390/jcm9020538 Medline

59. S. A. Lauer, K. H. Grantz, Q. Bi, F. K. Jones, Q. Zheng, H. R. Meredith, A. S. Azman, N. G. Reich, J. Lessler, The Incubation Period of Coronavirus Disease 2019 (COVID-19) From Publicly Reported Confirmed Cases: Estimation and Application. Ann. Intern. Med. 172, 577-582 (2020). doi:10.7326/M20-0504 Medline

60. Q. Bi, Y. Wu, S. Mei, C. Ye, X. Zou, Z. Zhang, X. Liu, L. Wei, S. A. Truelove, T. Zhang, W. Gao, C. Cheng, X. Tang, X. Wu, Y. Wu, B. Sun, S. Huang, Y. Sun, J. Zhang, T. Ma, J. Lessler, T. Feng, Epidemiology and transmission of COVID-19 in 391 cases and 1286 of their close contacts in Shenzhen, China: A retrospective cohort study. Lancet Infect. Dis. 0, S1473-3099(20)30287-5 (2020). doi:10.1016/S14733099(20)30287-5 Medline

61. H. Salje, C. Tran Kiem, N. Lefrancq, N. Courtejoie, P. Bosetti, J. Paireau, A. Andronico, N. Hozé, J. Richet, C.-L. Dubost, Y. Le Strat, J. Lessler, D. Levy-Bruhl, A. Fontanet, L. Opatowski, P.-Y. Boelle, S. Cauchemez, Estimating the burden of SARS-CoV-2 in France. Science eabc3517 (2020). doi:10.1126/science.abc3517 Medline

\section{ACKNOWLEDGMENTS}

We are grateful for the constructive feedback from those that reviewed a preprint of a previous version of this manuscript that was posted on 26 $6^{\text {th }}$ March 2020. Funding: CW is supported by a UK Medical Research Council Doctoral Training Programme studentship. PW is supported by an Imperial College Junior 
Research Fellowship. This work was supported by a grant from the Wellcome Trust and Centre funding from the UK Medical Research Council, both under a concordat with the UK Department for International Development. We additionally acknowledge support from Community Jameel and the NIHR Health Protection Research Unit in Modelling Methodology. Author contributions:

PGTW, CW, NMF and ACG conceived the study. PGTW, CW and OJW undertook the modelling and data analysis with input from MB, PW, AH and BD. ZC, DOM, WG and HT provided additional input into the framing of the results. SN and DGL provided clinical input into the model structure and parameterisation. ACG,

PGTW and CW produced the first draft of the manuscript. All authors contributed to the final draft. Competing interests: Dr Sabine van Elsland is also affiliated to the Department of Pediatrics and Child Health, Stellenbosch University,

Tygerberg Hospital, South Africa. Data and materials availability: All data used in this study can be freely downloaded from the cited sources. The model underlying the analyses is available in the form of an $\mathrm{R}$ package, available at https://github.com/mrc-ide/squire (v0.4.14 DOI: 10.5281/zenodo.3872340)

(34). The code used to generate these analyses is available at https:/github.com/mrc-ide/covid global_impact (DOI:

10.5281/zenodo.3872387). (35). This work is licensed under a Creative Commons Attribution 4.0 International (CC BY 4.0) license, which permits unrestricted use, distribution, and reproduction in any medium, provided the original work is properly cited. To view a copy of this license, visit

https://creativecommons.org/licenses/by/4.0/. This license does not apply to figures/photos/artwork or other content included in the article that is credited to a third party; obtain authorization from the rights holder before using such material.

\section{SUPPLEMENTARY MATERIALS}

science.sciencemag.org/cgi/content/full/science.abc0035/DC1

Materials and Methods

Figs. S1 to S6

Tables S1 to S5

References (36-61)

MDAR Reproducibility Checklist

Data S1

1 April 2020; accepted 9 June 2020

Published online 12 June 2020

10.1126/science.abc0035 
A

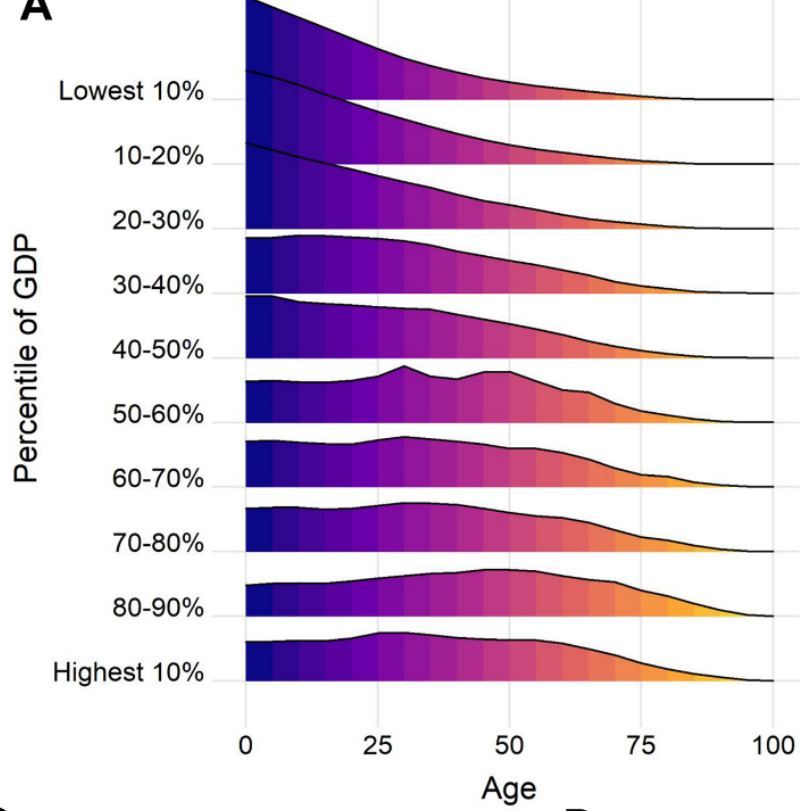

C

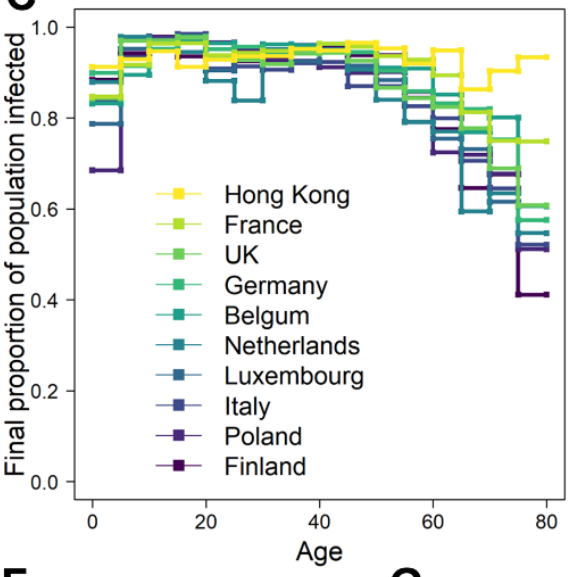

$\mathbf{F}$
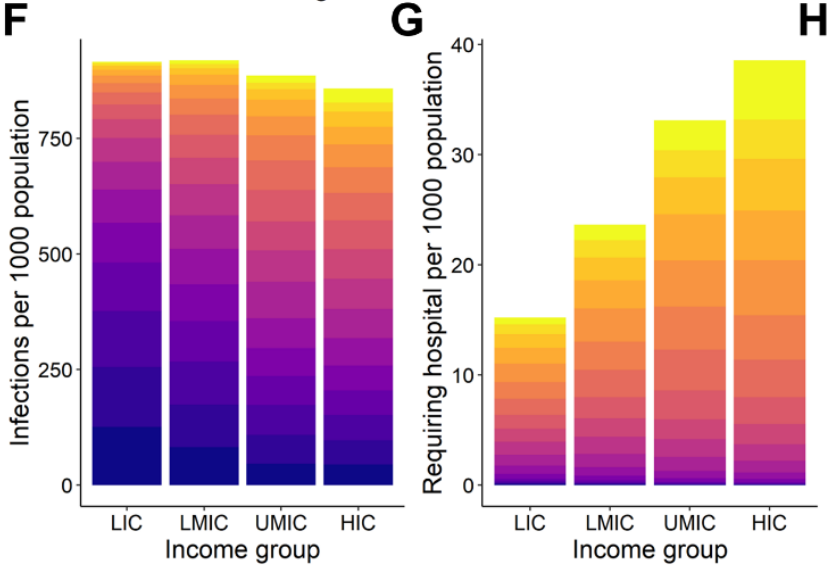

D

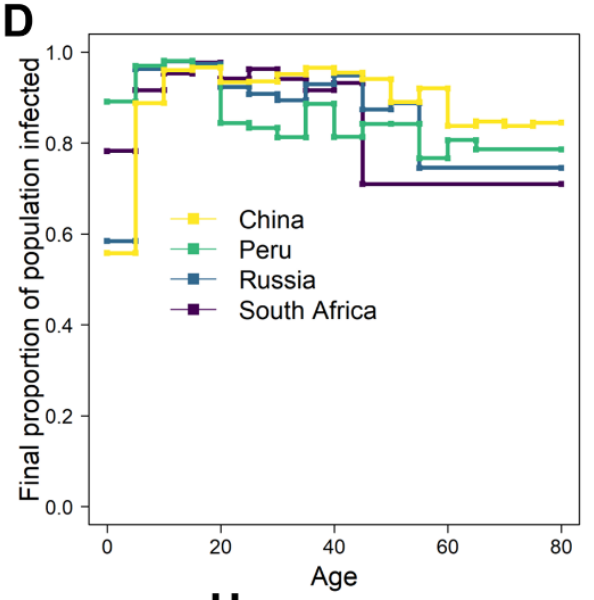

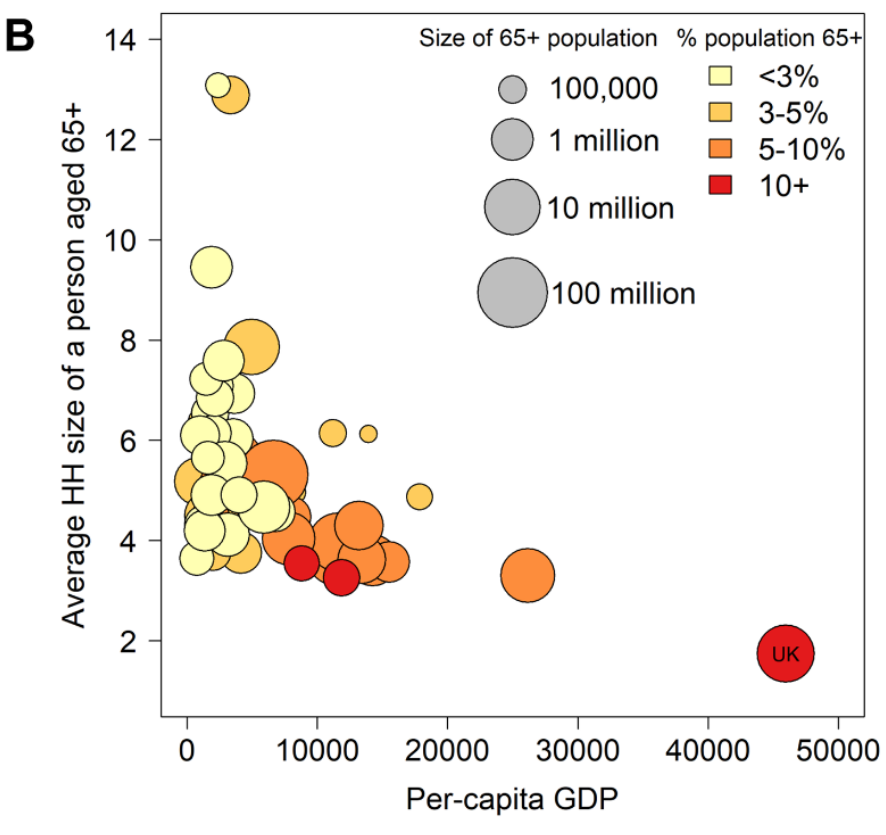

E

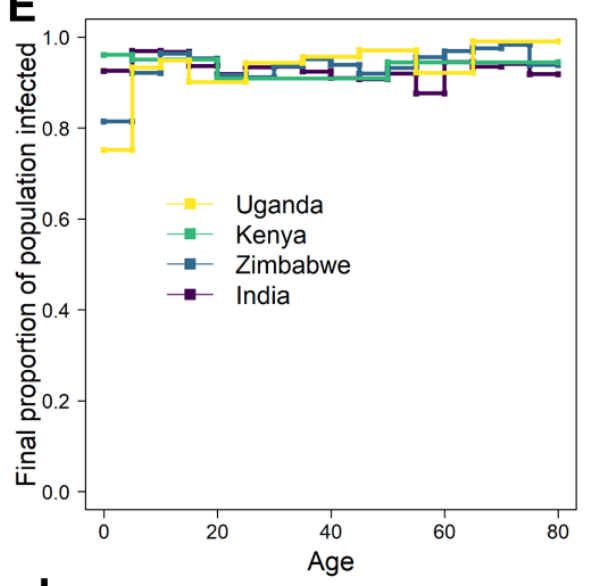

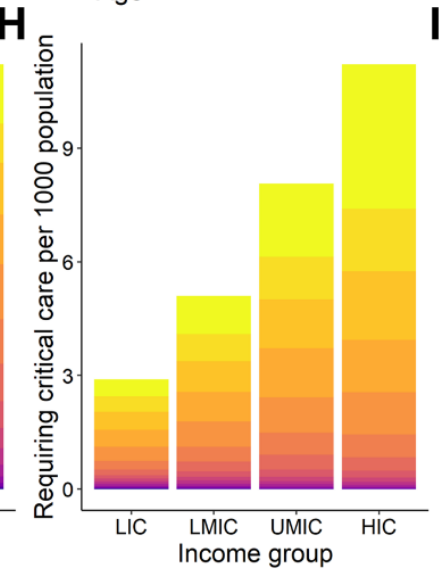

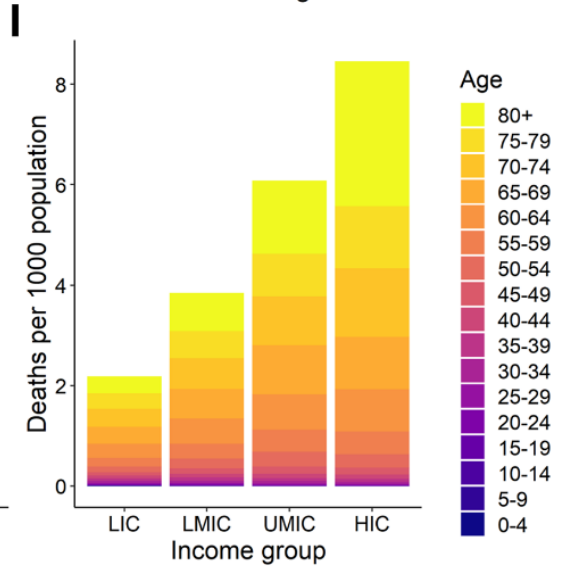


Fig. 1. Demographic, societal and mixing patterns relevant to SARS-CoV-2 transmission and burden. (A) Aggregated demographic patterns within 2020 World Population Prospects (WPP) projections across countries within each 2018 World Bank (WB) GDP per-capita decile. (B) Average household size within Demographic Health Surveys (DHS) of individuals aged 65 and over by 2018 WB GDP per-capita. For reference, the average household size of contacts in the UK is also provided as an example for a HIC. (C) Final proportion of population infected in an unmitigated epidemic for an age-structured SIR model with $\mathrm{R}_{0}=3.0$ and age-specific social mixing based upon contact surveys identified in HICs. (D) and (E) equivalent figure for surveys identified in UMICs and LMIC/LICs respectively. F-I output from simulations across countries of an unmitigated pandemic with $\mathrm{R}_{0}=3.0$. (F) shows the attack rate in terms of number of individuals infected per 1000 population, (G) the equivalent rates of infection leading to illness requiring hospitalization, $(\mathrm{H})$ illness requiring critical care and $(\mathrm{I})$ mortality assuming a health system functioning at the level of China throughout the pandemic. LIC=low income country, $\mathrm{LMIC}=$ low-middle income country, $\mathrm{UMIC}=$ upper-middle income country, $\mathrm{HIC}=$ high income country. 
A

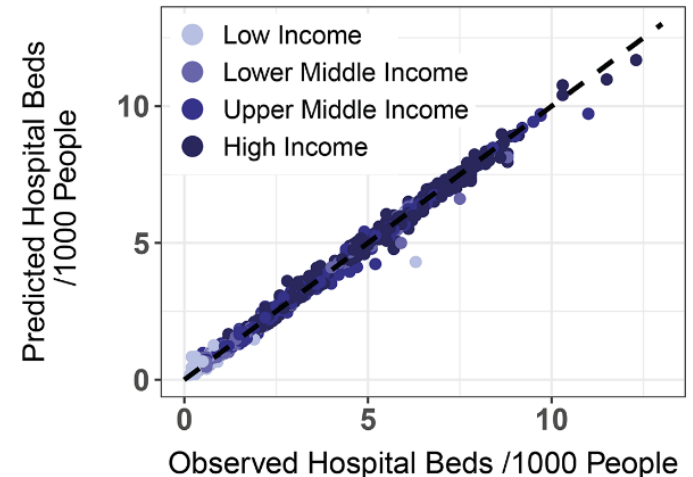

C

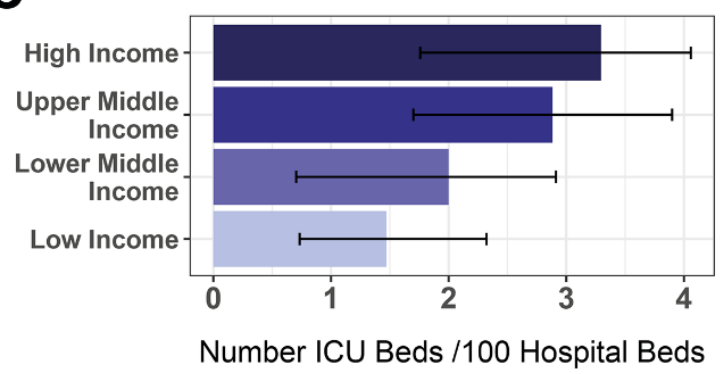

D

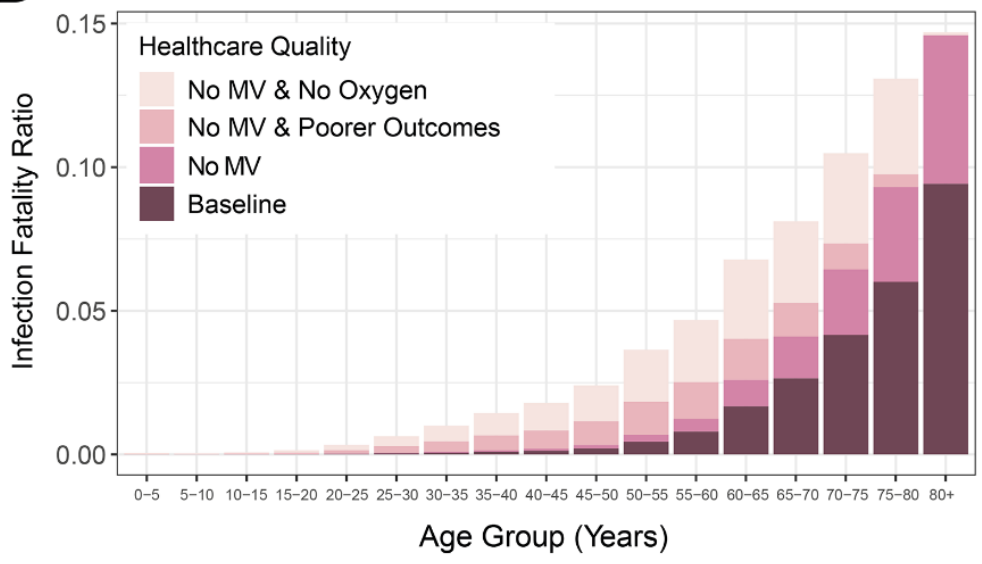

B

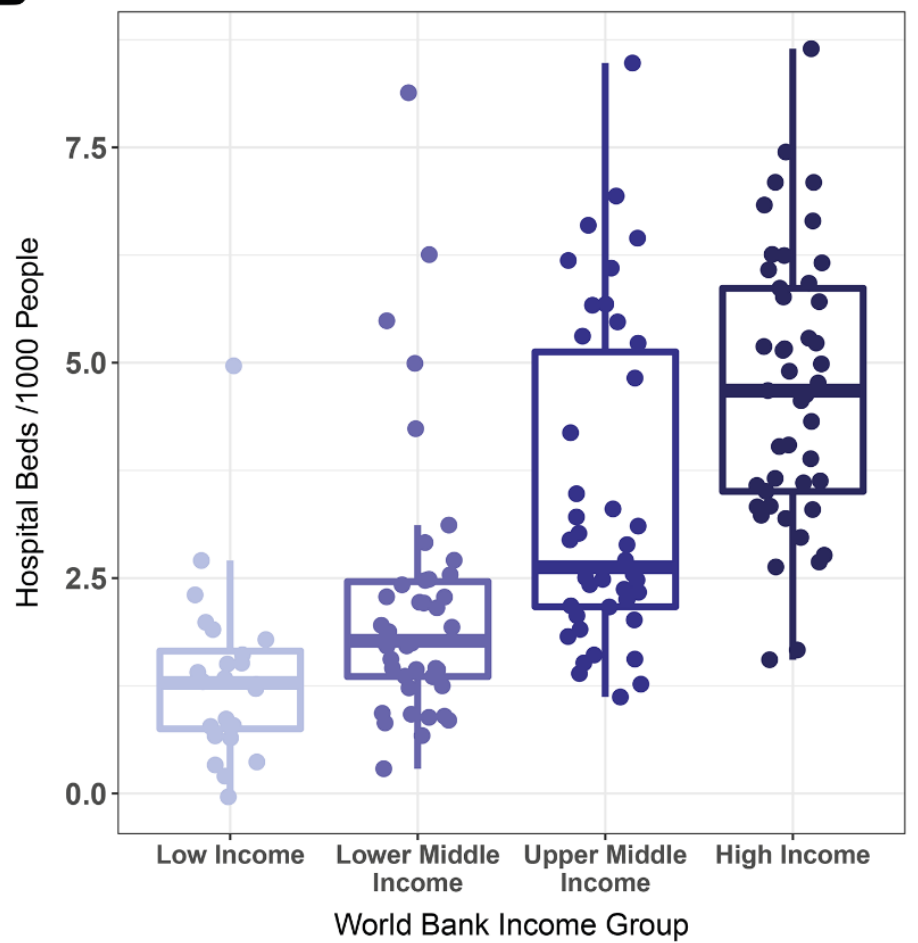

E

$\begin{array}{llll}\text { Baseline No MV } \quad \begin{array}{l}\text { No MV \& } \\ \text { Poorer } \\ \text { Outcomes }\end{array} & \begin{array}{l}\text { No MV \& } \\ \text { No Oxygen }\end{array}\end{array}$

LIC

0.24

0.38

0.68

1.17

LMIC

0.39

0.63

0.99

1.63

UMIC

0.61

0.98

2.24

HIC

1.03

1.66

3.34 
Fig. 2. Estimates of hospital bed and ICU capacity, and the potential impact of health care quality on the infection fatality ratio. (A) Comparison of BRT model prediction and empirically observed numbers of hospital beds per 1000 population. Each point represents a country, with the x-axis indicating the observed number of hospital beds per 1000 population for that country, and the $y$-axis indicating the model predicted number of hospital beds per 1000 population. Coloring of the points indicates which World Bank income strata the country belongs to. (B) Boxplots of the number of hospital beds per 1000 population, stratified by World Bank income group. Points are modeled estimates of hospital beds per 1,000 population obtained from the model. (C) Results from a systematic review describing the percentage of all hospital beds that are in ICUs, stratified by World Bank income group. Error bars indicate the interquartile range of the median. (D) Age-stratified scenarios for the infection fatality ratio under different health care quality. The baseline are estimates based on data for highincome settings. "No MV" denotes not being able to access an ICU unit with mechanical ventilation available. "Poorer outcomes" represents a higher risk of mortality from severe pneumonia in an LMIC setting if only limited or poor-quality oxygen support is available. "No Oxygen" represents the outcomes if hospitalized patients do not receive oxygen support. The stacked bars represent the cumulative increase in IFR at each stage. Note that the final stage "No MV and No Oxygen" represents the additional IFR due to increasing mortality rates from 20\% in the presence of limited/poor-quality oxygen support to $60 \%$ in the absence of any oxygen support. (E) Estimated representative IFR averaged across age-groups in different settings under a range of health care quality assumptions. The differences between LIC, LMIC, UMIC and HIC at baseline reflect the demography and social contact patterns but otherwise assume the same health care quality. Lower health care quality is not shown for UMIC and HIC as these settings are likely to have the quality of health care incorporated in the baseline estimates. 
A

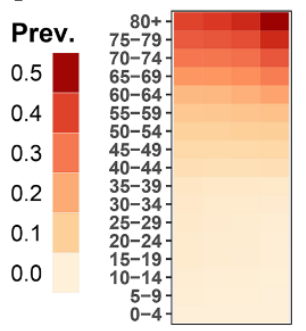

B

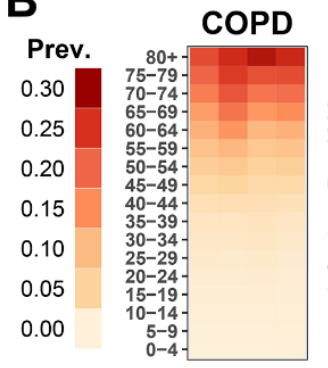

C

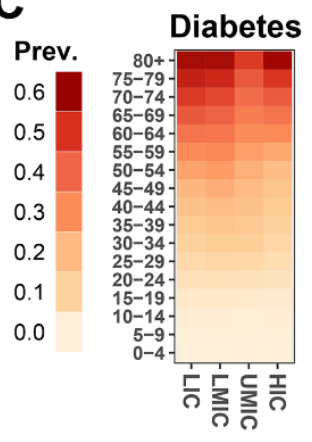

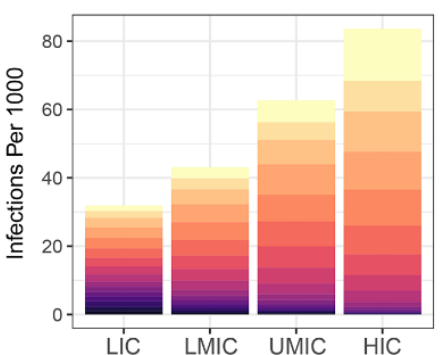

LIC LMIC UMIC HIC

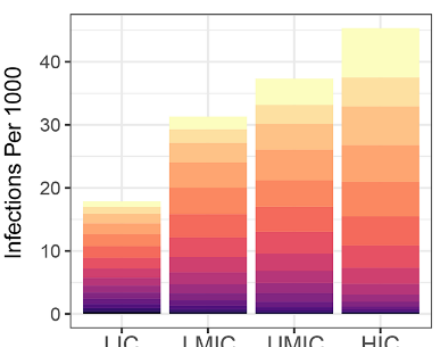

E

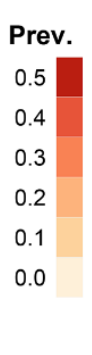

F

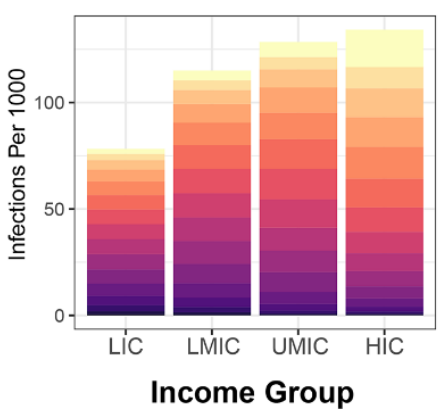

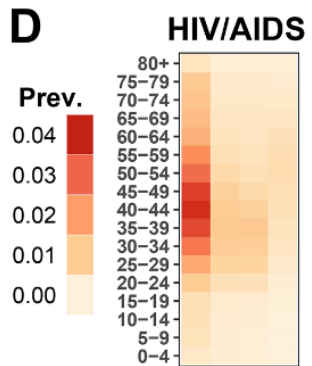

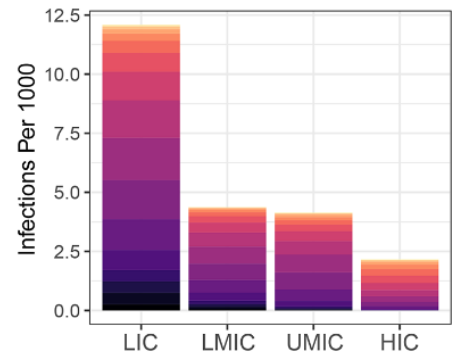

Age Group

$80+$

$75-79$

70-74

65-69

Malnutrition
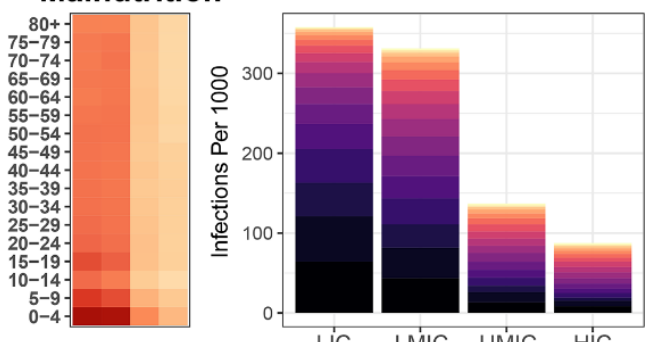

60-64

55-59

$50-54$

45-49

40-44

35-39

30-34

25-29

20-24

15-19

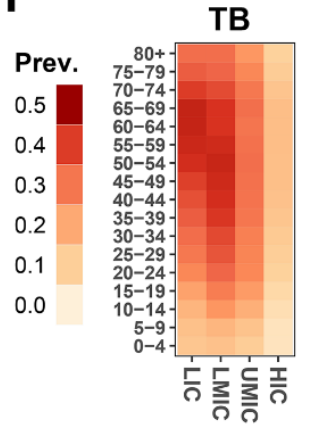

10-14

5-9

0-4

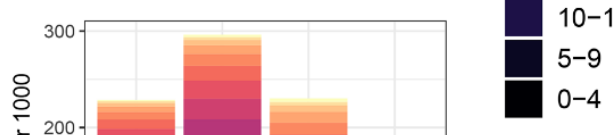

Fig. 3. The prevalence of different co-morbidities across income settings and the proportion of SARS-CoV-2 infections co-occurring with them. The age-distribution of co-morbidities relevant as modifiers of COVID-19 disease severity was extracted from Global Burden of Disease 2017 estimates (12) and integrated with estimates of the predicted age-distribution of infection in an unmitigated pandemic scenario. For (A) Cardiovascular Disease, (B) Chronic Obstructive Pulmonary Disease, (C) Diabetes, (D) HIV/AIDS, (E) Malnutrition and (F) Tuberculosis, the left heatmap shows the age-distribution of these co-morbidities across different incomesettings, expressed as the proportion of the population in that income setting that have the comorbidity. The bar charts (colored according to age-group) shown the number of infections per 1,000 population that co-occur with the respective co-morbidity. 
A
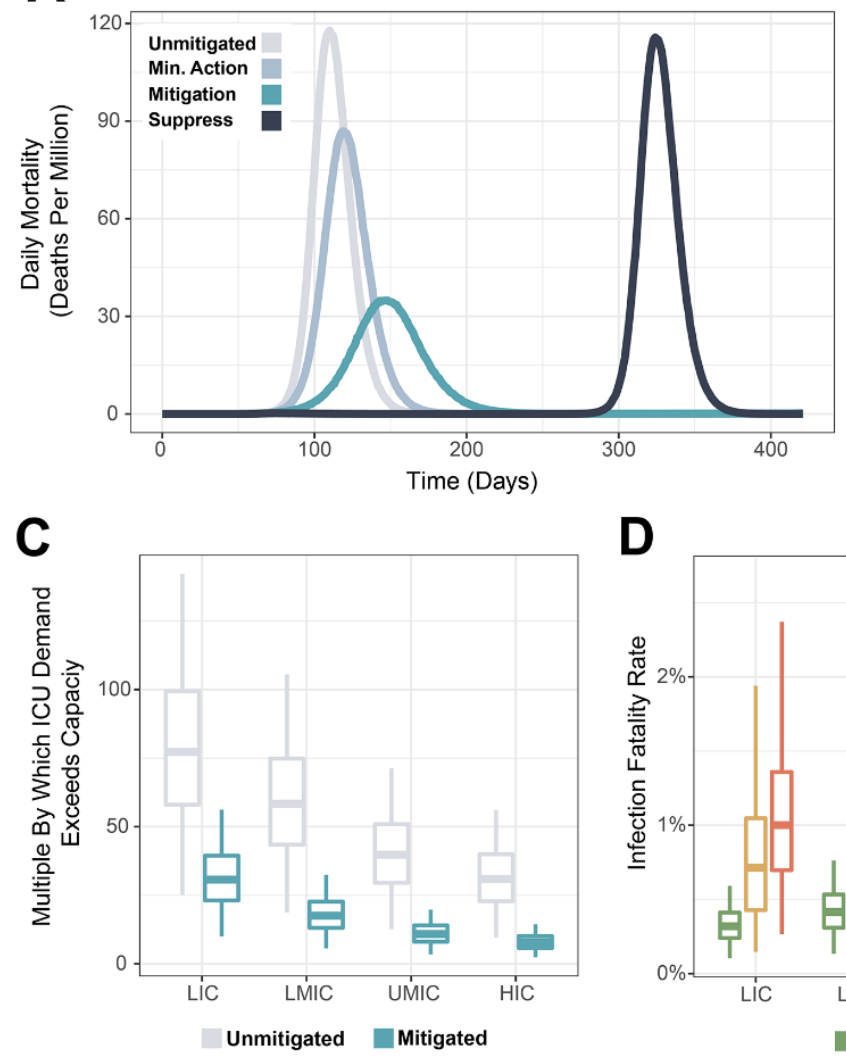

B

D

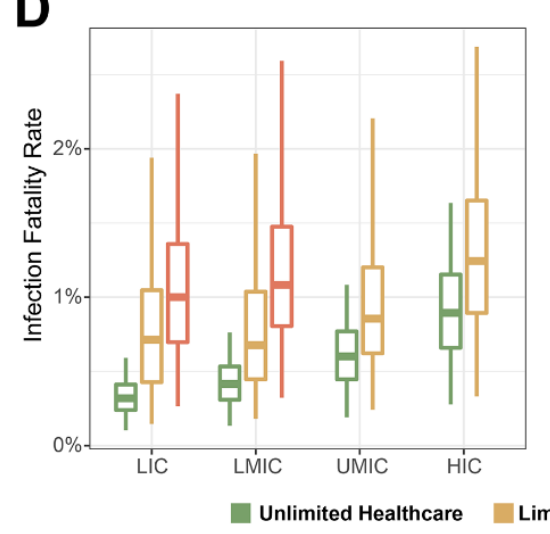

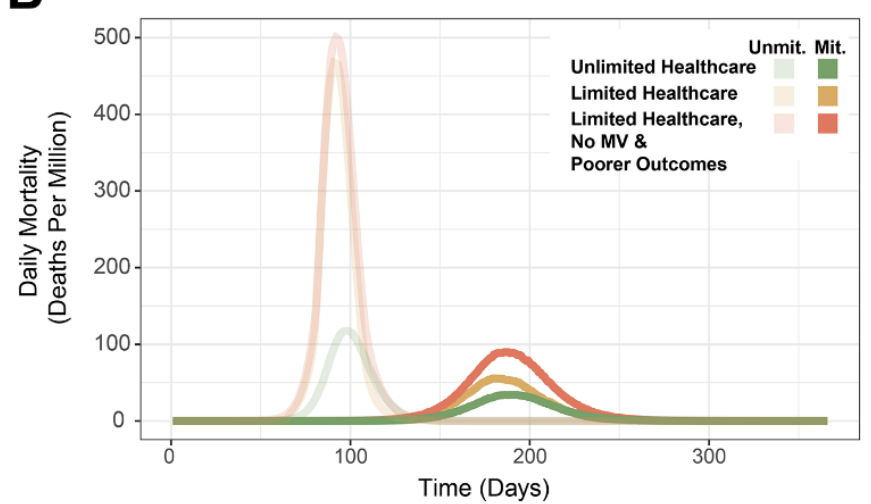

$\mathbf{E}$

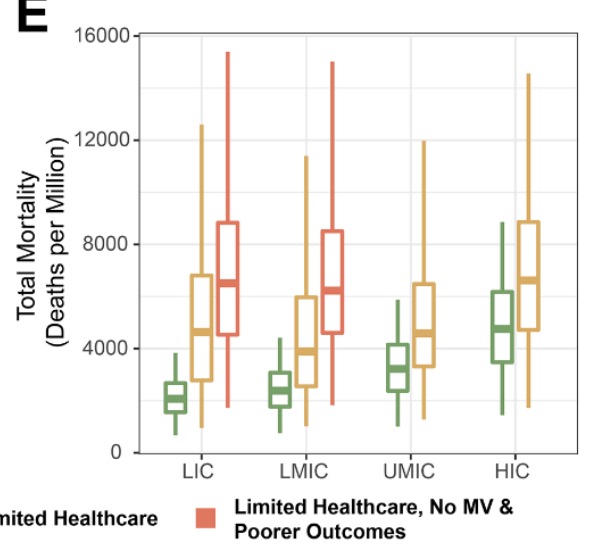

Fig. 4. The impact of health care capacity and quality on COVID-19 mortality in different settings (A) Representative epidemic trajectories for an unmitigated epidemic (grey line), an epidemic involving minimal social distancing (pale blue line, $20 \%$ reduction in social contacts), an epidemic involving extensive social distancing (teal, 45\% reduction in social contacts) and an epidemic trajectory that involves extensive suppression (75\% reduction in social contacts) followed by lift after 6 months, leading to resurgence (dark blue line). (B) The excess deaths associated with constraints on health care quality and quantity, including the deaths associated with a hypothetical setting with unlimited high quality health care (green lines), settings where high quality health care is available but limited (yellow lines) and where only limited, poorer quality health care is available (orange lines). Pale lines show an unmitigated scenario, colored lines a mitigated scenario. (C) The multiple by which ICU demand exceeds capacity for each World Bank income strata for an unmitigated (grey) and mitigated (teal) epidemic. (D) The modeled IFR for different World Bank income strata under different scenarios of health care quality and quantity available, assuming a mitigated scenario in which baseline contacts are reduced by $45 \%$. (E) The modeled deaths per million population for different World Bank income strata under different assumptions of health care quality and quantity available, assuming a mitigated scenario in which baseline contacts are reduced by $45 \%$. Plots show medians (bars) and interquartile ranges (boxes), as well as points $<1.5 x$ the IQR (whiskers) and $>1.5 x$ (points) from 500 parameter draws. 
A
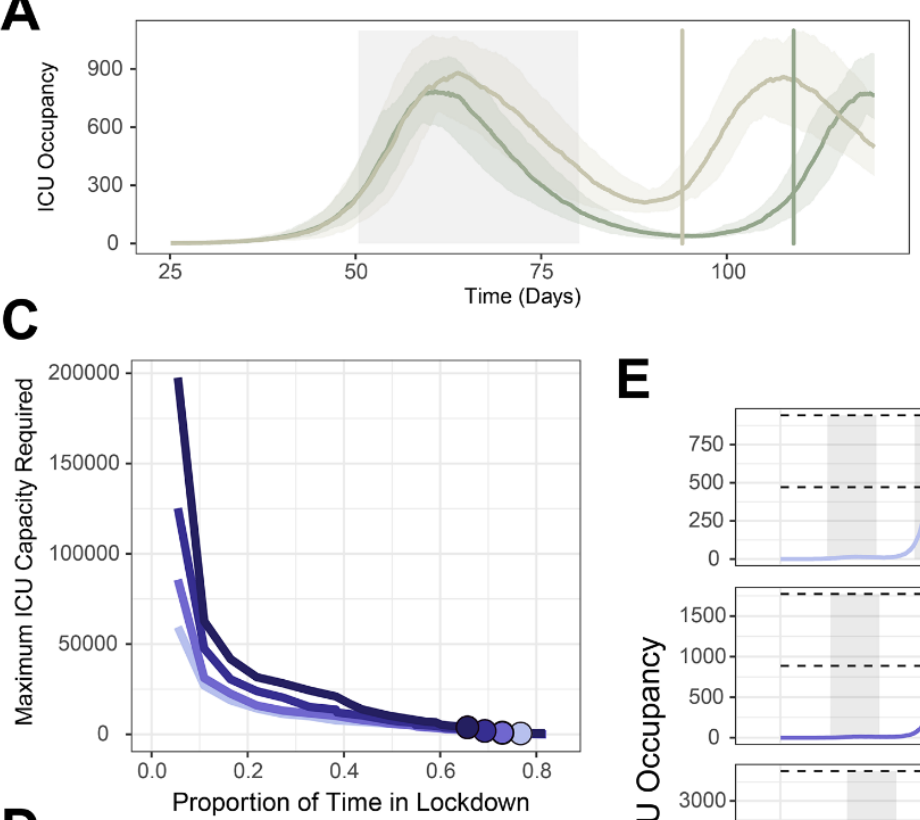

D

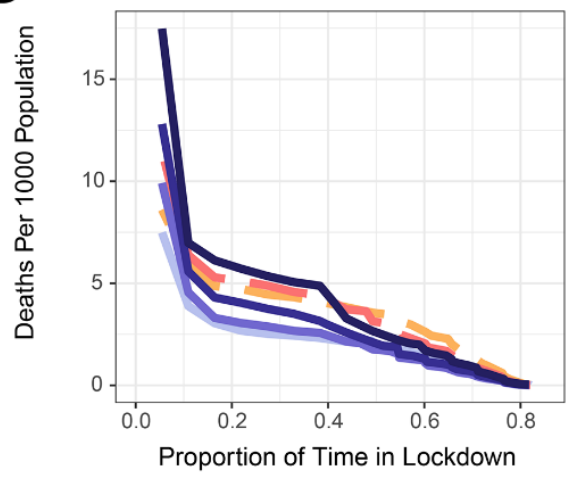

$\mathbf{E}$
B

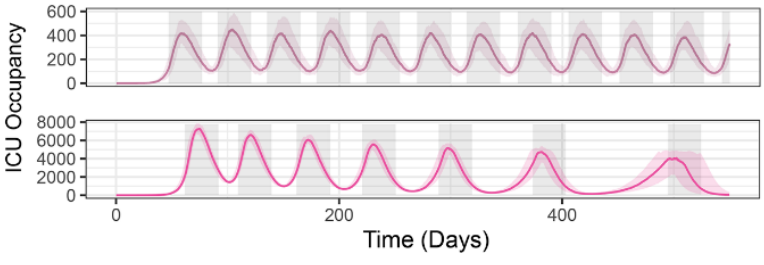

Low Trigger Threshold
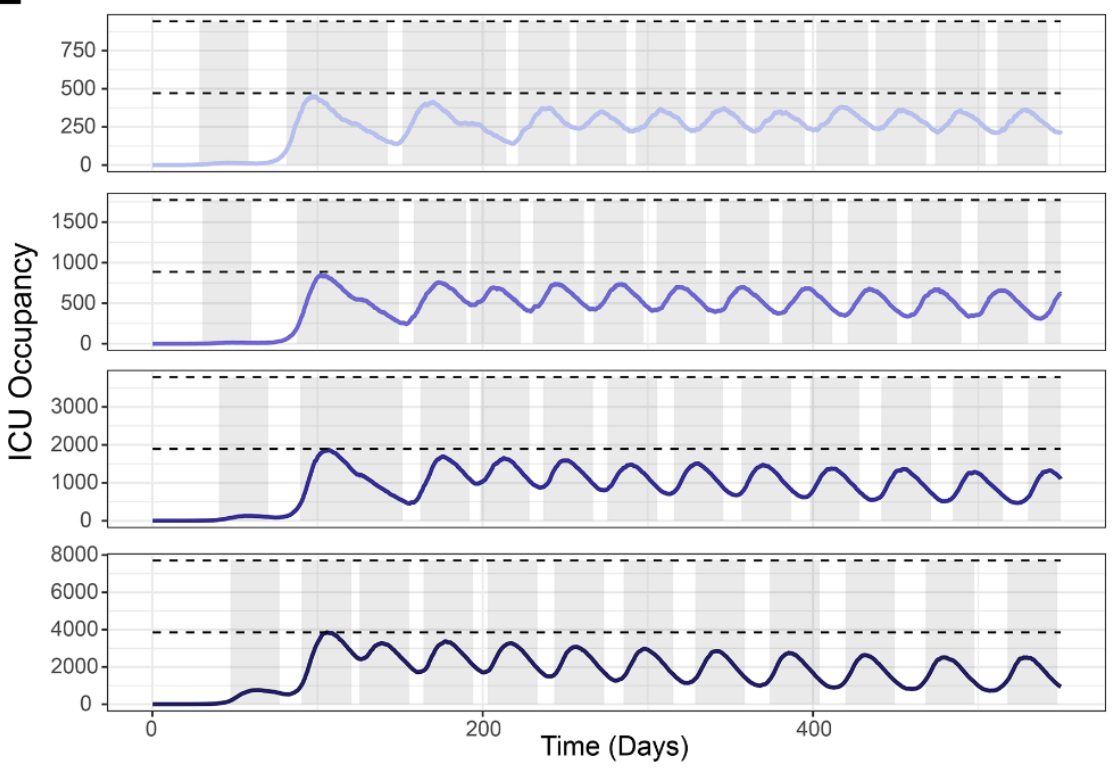

Low Income Lower-Middle Income

Low Income Poorer Outcomes
High Income

Lower-Middle Income Poorer Outcomes

Fig. 5. The proportion of time that countries will need to spend in lockdown in order to remain within healthsystem critical care capacity. Scenarios are generated using the stochastic SEIR model (see Materials and Methods) (A) The time period between lockdowns for a representative LIC setting, and how it varies with the extent of suppression during lockdown. Grey shaded area denotes time period of first suppression (triggered at a threshold of 60 ICU cases per day) and then brown (75\% reduction) and green (85\% reduction) vertical lines indicate the next timepoint at which suppression would be implemented (using the same threshold). (B) Time under suppression over the next 18 months for triggering thresholds of 30 (pale pink) and 500 (brighter pink) ICU cases per day respectively. Grey shaded areas indicate time in suppression (a 75\% reduction in $\mathrm{R}_{0}$ ). (C) The proportion of time required to be spent in lockdown over the next 18 months as a function of the maximum ICU demand for representative LIC, LMIC, UMIC and HIC (colored purple lines). Colored points indicate the median ICU capacity for each of these different income strata. (D) The proportion of time required to be spent in lockdown over the next 18 months as a function of the number of deaths caused by the COVID-19 epidemic for representative LIC, LMIC, UMIC and HIC assuming comparable quality (but not quantity) of health care across all settings (colored purple lines), and when assuming a reduction in the quality of health care available in LICs and LMICs (red and orange dashed lines respectively). (E) Modeled COVID-19 epidemic trajectories over the next 18 months for representative LIC, LMIC, UMIC and HIC where suppression is implemented at ICU incidence trigger thresholds in order to keep the maximum ICU demand beneath 50\% of ICU capacity. Note that the first triggering of suppression has been determined based on the actual patterns of suppression timing observed across LICs, LMICs, UMICs and HICs. 
Table 1. Estimated stage of the epidemic at suppression across regions and income strata and ratios of reported cases to deaths prior to suppression.

\begin{tabular}{|c|c|c|c|c|c|c|}
\hline & $\begin{array}{l}\text { Countries } \\
\text { initiating } \\
\text { suppression } \\
\text { measures }\end{array}$ & $\begin{array}{l}\text { Median date } \\
\text { suppression } \\
\text { implemented } \\
\text { (range) }\end{array}$ & $\begin{array}{c}\text { Median Cases/million } \\
\text { prior to suppression } \\
\text { (range) }\end{array}$ & $\begin{array}{l}\text { Median Deaths/million } \\
\text { prior to suppression } \\
\text { (range) }\end{array}$ & $\begin{array}{c}\text { With }>=3 \\
\text { deaths prior to } \\
\text { suppression }\end{array}$ & $\begin{array}{l}\text { Median ratio of } \\
\text { cases to deaths }\end{array}$ \\
\hline Worldwide & 121 & 24/03 (08/03-19/04) & 6.01 (0 -449.24) & $0(0-10.447)$ & 50 & $45.8(5.1-325.2)$ \\
\hline \multicolumn{7}{|c|}{ Region } \\
\hline $\begin{array}{l}\text { East Asia \& } \\
\text { Pacific }\end{array}$ & 10 & $25 / 03(14 / 03-10 / 04)$ & $3.08(0-149.37)$ & $0(0-0.549)$ & 9 & $47.3(9-325.2)$ \\
\hline $\begin{array}{l}\text { Europe \& } \\
\text { Central Asia }\end{array}$ & 34 & 22/03 (08/03- 03/04) & 72.08 (0.07-449.24) & $0.340(0-10.447)$ & 20 & $46.7(9-320.3)$ \\
\hline $\begin{array}{l}\text { Latin America } \\
\text { \& Caribbean }\end{array}$ & 21 & 22/03 (16/03- 08/04) & $6.01(0.18-156.59)$ & $0(0-0.393)$ & 6 & $63.9(30.2-102)$ \\
\hline Middle East \& & & & & & & \\
\hline North Africa & 14 & 23/03 (17/03 - 30/03) & $12.44(0-353.31)$ & $0(0-1.763)$ & 6 & 16.5 (5.1 - 139.7) \\
\hline North America & 0 & NA & NA & NA & 2 & $43.5(14.5-72.6)$ \\
\hline South Asia & 4 & 26/03 (20/03-02/04) & $2.41(0.07-35.15)$ & $0(0-0.051)$ & 1 & 80 (NA) \\
\hline $\begin{array}{l}\text { Sub-Saharan } \\
\text { Africa }\end{array}$ & 38 & $28 / 03(21 / 03-19 / 04)$ & $0.92(0-101.68)$ & $0.002(0-1.799)$ & 5 & $10.7(7.8-63.6)$ \\
\hline \multicolumn{7}{|c|}{ Income Strata } \\
\hline Low income & 23 & 28/03 (20/03-19/04) & $0.57(0-6.13)$ & $0(0-0.791)$ & 3 & $8.6(7.8-10.7)$ \\
\hline $\begin{array}{l}\text { Lower middle } \\
\text { income }\end{array}$ & 22 & 25/03 (14/03-10/04) & $0.58(0.05-30.99)$ & 0 (0-1.799) & 7 & $19.3(9-80)$ \\
\hline $\begin{array}{l}\text { Upper middle } \\
\text { income }\end{array}$ & 41 & 24/03 (16/03- 08/04) & $9.98(0-135.34)$ & $0.044(0-3.548)$ & 13 & $38.8(5.1-191.8)$ \\
\hline High income & 35 & 23/03 (08/03-08/04) & $97.30(5.14-449.24)$ & $0.202(0-10.447)$ & 27 & $72.6(9-325.2)$ \\
\hline
\end{tabular}

${ }^{\text {x}}$ Does not include countries implementing suppression not listed in ACAP data as of April $17^{\text {th }} 2020$

${ }^{6}$ Includes countries who have not yet implemented suppression in ACAP data as of April $17^{\text {th }} 2020$ where infections are assumed to have grown exponentially until the date of most recent measure reported in ACAP. For countries excluded from suppression analysis (Brazil, China, Iran, Japan, Singapore, South Korea and the United States of America) analysis was restricted to the time prior to 10 cumulative deaths reported. 